\title{
Abstracts
}

\section{Psychosocial/Quality of Life}

\author{
Sexual Identity and Orientation in Adult Men and \\ Women with Spina Bifida \\ Szymanski, Konrad ${ }^{1}$, Hensel, Devon ${ }^{2}$, Wiener, \\ John S. ${ }^{3}$, Whittam, Benjamin ${ }^{1}$, Cain, Mark ${ }^{1}$, Misseri, \\ Rosalia $^{1}$ \\ ${ }^{1}$ Riley Hospital for Children \\ ${ }^{2}$ Department of Pediatrics and Department of Sociol- \\ ogy, Indiana University Purdue University Indianapo- \\ lis \\ ${ }^{3}$ Section of Pediatric Urology, Duke University Medi- \\ cal Center
}

Background While Spina Bifida (SB) affects multiple organ systems, sexuality has received little attention. We aimed to determine sexual identity and orientation in adults with SB.

Methods We administered an international online survey to men and women $\geqslant 18$ years old with $\mathrm{SB}$ over a 6-month period. Participants were recruited at SB clinics and through local, national and international SB organizations via social media. We collected data on demographics, surgeries, sexual identity and orientation. Non-parametric tests were used for statistical analysis. Results The median age of 69 men and 106 women participating was 35 years old (IQR: 29-43, 54.3\% shunted, $46.3 \%$ community ambulators). Overall, $73.7 \%$ had some post-secondary education and $40.0 \%$ lived outside the United States. Most commonly, men identified themselves as male $(95.7 \%)$, while $1.4 \%$ described themselves as female, $1.4 \%$ as transgender and $1.4 \%$ as other. All women who reported their sexual identity identified as female $(99.1 \%)$, with $0.9 \%$ not providing an answer. Differences in reporting a sexual identity different from biological gender between men and women did not reach statistical significance $(p=0.06)$. Most men reported a heterosexual orientation (91.3\%), followed by gay (7.3\%) and bisexual $(1.5 \%)$. Most women reported a heterosexual orientation $(84.9 \%)$, followed by bisexual (10.4\%), lesbian
(1.9\%) and other (2.8\%). Men were more likely to report gay orientation while women more likely to report bisexual orientation $(p=0.01)$.

Conclusions As in the general population, sexual identity typically coincides with biological gender. The sexual orientation of adults with SB appears to mirror the general population based on national statistics.

\section{Patient and Parental Stress with Spina Bifida Kongkasuwan, Ratcharin ${ }^{1}$, Blanchard, Alesia ${ }^{1,2}$, Simpson, Tess ${ }^{1}$, O'Keeffe, Shannon ${ }^{1,2}$, Clayton, Jerry ${ }^{1,2}$, Wilson, Pamela ${ }^{1,2}$ \\ ${ }^{1}$ Children's hospital Colorado \\ ${ }^{2}$ University of Colorado, School of Medicine}

Background Spina Bifida is a neural tube defect negatively affecting spine and spinal cord development. This complex physical disability in children can lead to caregiver burden (parental stress, patient anxiety or depression).

Methods We retrospectively analyzed 171 Spina Bifida patients from the spinal defects clinic at Children's Hospital Colorado seen between 01/01/14 to 09/30/16. This clinic utilizes the Parental Stress Score (PSS; 18 90), the Patient Health Questionnaires (PHQ9; 0-27) and the Generalized Anxiety Disorder (GAD7; 0-21) for patients $\geqslant 12$. These data were used to evaluate parental stress, patient depression and anxiety. Associations between parental stress, age, race, ethnicity, gender, health care system, lesion level, diagnosis, ambulation status, shunt status, bladder, and bowel problems were conducted using SPSS.

Results The mean PSS score was 32 (18-59; SD = 9.01) which showed statistically significant correlations with patient's age $(p=0.022 ; r=0.175)$ and ambulation status $(p=0.042)$. The mean patient PHQ9 score was $2.5(0-22 ; \mathrm{SD}=4.31)$ with $17.2 \%$ of the patients reaching criteria for depression. Three patients 
indicated having suicidal ideation also scored high on the GAD7 and PSS evaluations. Criteria for anxiety on the GAD7 was met by $29.7 \%$ of the population (mean $=3.68 ; 0-21$; SD = 4.94). Higher PSS scores correlated well with PHQ9 scores $(r=0.376)$ and GAD7 scores $(r=0.463)$.

Conclusions Patient Anxiety and depression significantly correlated with parental stress especially if suicidal ideation was reported by their children. Older age and limited functional ability to ambulate appear to associate with parental stress. We found slightly elevated rates of anxiety and depression in the Spina Bifida population at our clinic. Possible explanations for these results include unforeseen impacts of concurrent care and neuromuscular changes. Limitations of this study include sample size and time between surveys.

\section{Subjective Quality of Life for Youth with Spina Bifida \\ McDougall, Janette $\mathrm{e}^{1,2,3}$, Wright, Virginia ${ }^{3,4}$ \\ ${ }^{1}$ Thames Valley Children's Centre \\ ${ }^{2}$ Western University \\ ${ }^{3}$ Bloorview Research Institute \\ ${ }^{4}$ University of Toronto}

Background This research examined the subjective QOL of youth with Spina Bifida (SB) by: a) comparing subjective QOL scores of these youth with the scores of youth with other chronic conditions; b) comparing youth subjective QOL mean scores as reported by themselves and by one of their parents; and c) exploring cognitive and physical functioning as correlates of youths' subjective QOL, controlling for youth age, gender, and income.

Methods Thirty-six youth aged 11 to 17 years with SB participated. Youth were recruited from rehabilitation centres in Ontario, Canada. Both youth and parents completed questionnaires. Subjective QOL was measured using the Student Life Satisfaction Scale (SLSS). The SLSS includes 5 items on a 6-point Likert scale. Analysis of variance examined mean differences in scores among youth with different conditions. A $t$-test assessed mean differences in youth and parent scores. Linear regression identified correlates of subjective youth QOL.

Results No significant differences $(F=1.35 ; p<$ 0.22 ) existed regarding subjective QOL scores among youth by chronic condition. The group mean for youth with SB $(M=24.75, S D=4.55, n=36)$ indicated that, on average, they 'mildly agree' their life is go- ing well. Youth scored significantly higher than parents $(t=2.97 ; p<0.006)$ regarding their subjective QOL $(M=24.59, S D=4.62, n=34 ; M=21.94, S D=$ $4.67, n=34$, respectively). Cognitive functioning was significantly and negatively related to youth subjective QOL $(t=-3.35, p<0.003)$. Physical functioning was not a significant correlate.

Conclusions Given that, on average, youth with SB only mildly agree their life is going well, it is important for their subjective QOL to be assessed. It is also beneficial to assess both youth and parent perspectives, as each offer unique insights. Cognitive functioning was negatively associated with youths' subjective QOL, emphasizing the importance of examining cognition of youth with SB, in addition to physical status.

\section{Discrepancies in Quality of Life Reported by People with Spina Bifida and their Caregivers Szymanski, Konrad, Cain, Mark, Misseri, Rosalia Riley Hospital for Children}

Background Caregivers may not appropriately assess health-related quality of life (HRQOL) of people with Spina Bifida (SB). Our aim was to identify clinically significant differences between SB patient- and caregiver-reported HRQOL and to determine factors are associated with caregivers under/overestimating HRQOL.

Methods An international group of children ( $\geqslant 8$ yo) and adults with SB and their caregivers were surveyed. Demographics and caregiver type (mother, father, other) were assessed. Patients and caregivers reported SB-specific HRQOL using validated QUALAS instruments. Analyses involved the Bowel/Bladder domain common to all age groups (primary) and 4 other age-specific domains (secondary). Clinically significant differences between HRQOL reports were based on published thresholds. Patient-caregiver reliability was assessed with kappa coefficients (fair: 0.21-0.40, moderate: 0.41-0.60). Fisher's exact test and logistic regression were used in analysis.

Results Among 329 patient-caregiver pairs, median patient age was 15 (range $8-53,51.8 \%$ male) and $73.0 \%$ of caregivers were mothers. Overall, there was only slight patient-caregiver correlation for Bladder/Bowel domain $(k=0.15)$. Caregivers reported HRQOL clinically different from patient-reports $42.2 \%$ of the time, under/overestimating HRQOL at similar rates $(23.1 \%$ vs. $19.2 \%, p=0.20)$. Patient- and caregiver-reported HRQOL had only slight correlation 
for remaining QUALAS domains ( $k=0.05-0.15)$, with caregivers misreporting HRQOL 34.9-55.6\% of the time. On multivariate analysis, no caregiver type estimated patient-reported HRQOL more accurately for any QUALAS domain $(p \geqslant 0.10)$, whether or not they lived with the patient $(p \geqslant 0.26)$.

Conclusions Almost half of the time, caregivers misreport HRQOL of people with SB to a degree that is clinically significant. There is no surrogate to self-reported HRQOL and, whenever possible, patients should report their own subjective outcomes.

\section{Pain and Spina Bifida: Common, Severe, and Worse with Age}

Szymanski, Konrad ${ }^{1}$, Misseri, Rosalia ${ }^{1}$, Cain, Mark ${ }^{1}$, Hirsh, Adam ${ }^{2}$

${ }^{1}$ Riley Hospital for Children

${ }^{2}$ Department of Psychology, Indiana University - Purdue University Indianapolis

Background Pain is an important clinical outcome and may be common in people with Spina Bifida (SB). Our aims were to determine the prevalence of pain in the SB population, its severity, and impact on everyday activities.

Methods An international cross-sectional study was conducted in a population with SB and controls $(\geqslant 8$ yo) recruited online via social media and at outpatient SB and pediatric clinics. Pain in the last 4 weeks was measured using Wong-Baker Faces scale (rating: 0-10, 10: worst pain). Impact of pain was assessed using a single item ("Did pain stop you from doing what you wanted to do?") rated on a Likert scale from 0 (never) to 100 (always). Fisher's exact tests, t-tests, Pearson's correlation (r), logistic and linear regression were used for analysis.

Results Eight hundred people with SB (mean age 23.6 yo, $44.5 \%$ children, $41.4 \%$ male, $67.3 \%$ shunted, $52.6 \%$ community ambulators) and 225 controls were included in the analysis (similar age, gender, $p \geqslant 0.76$ ). People with SB were more likely to report pain $(64.6 \%$ vs. $19.6 \%, p<0.0001)$ and more severe mean pain scores (4.1 vs. 3.0, $p=0.0002$ ). Increasing age was associated with higher prevalence of pain in the SB group $(61.7 \%$ in $8-12$ yo to $90.3 \%$ in $>50$ yo, $p<0.0001)$, but not in controls $(p=0.17)$. On multivariate analysis of the SB group, pain prevalence increased after 25 yo $(p=0.04)$, in females $(p=0.01)$, those with developmental delay $(p=0.01)$ and no shunt $(p=0.001)$. Higher pain scores correlated with higher pain impact for those with and without SB ( $r=0.61$ and 0.69 , both $p<0.0001)$, similar for both groups ( $p=0.054)$. On a multivariate analysis of the SB group, higher pain impact was associated with higher pain scores $(p<$ $0.0001)$, non-white race $(p=0.02)$ and developmental delay $(p=0.003)$.

Conclusions Pain affects the majority of the SB population. Its prevalence increases through adulthood. People with SB experience more severe pain than those without SB. More research is needed to improve their care.

\section{Parental Perceptions of Video Education for Spina Bifida in the Neonatal Intensive Care Unit and at Discharge}

Howell, Lori J.

Children's Hospital of Philadelphia

Background An estimated 3,000 pregnancies are affected by Spina Bifida (SB) annually resulting in 1,500 births. SB care is complex, multidisciplinary and requires long term follow-up. There is a paucity of information related to SB care in the Neonatal Intensive Care Unit (NICU). Most of the SB literature is descriptive in nature and does not focus on care in the NICU and at discharge. There is wide variability of SB care reported resulting in an inability to standardize parent education. Parents may not be getting the necessary education to take their baby home from the NICU and may have unclear expectations for the NICU stay. Although there are few studies to date, all support parental prenatal and postnatal education of parents for a variety of birth defects. However, SB education in the newborn period has not been investigated nor standardized.

Methods Evidence based project and IRB exempt study using a video intervention and survey tool distributed electronically to a convenience sample of parents whose child with SB was less than one year of age in a large, urban, children's hospital.

Results Surveys were analyzed using descriptive statistics rating the video highly positively in all categories. Primigravida, multigravida, NICU length of stay or level of SB lesion had no effect on parent rankings. Qualitative comments complemented the high survey scores.

Conclusions Findings from this project help to fill a gap in SB education and provide an opportunity to understand parent perceptions of their educational needs for a child with SB while in the NICU. Understand- 
ing these perceptions may result in improved SB care, decrease ambiguities, and improve professional performance. Further studies are needed to address prenatal parental educational needs, replicate this work with a larger sample size, multiple centers, and extend this work into other age groups.

\section{Use of a Gaming App to Promote Self-management of Spinal Cord Dysfunction}

Meade, Michelle A. University of Michigan

Background This presentation will describe the development and assessment of SCI Hard- a mobile gaming app designed to promote self-management skills among adolescents and young adults with Spinal Cord Injury and Dysfunction (SCI/D).

Methods A randomized control trial (RCT) design is being used to examine the effectiveness of SCI Hard in promoting self-management skills and health behaviors among adolescents with spinal cord dysfunction. After completing baseline assessments, participants are randomized to either SCI Hard or a placebo control game. Game play is verified before participants are allowed to continue with the study. Follow-up assessments will be completed at 1 month and 3 months post-baseline.

Results This is an active study. While the clinical trial will not be completed by the time of this conference, we will extract, summarize and present the data from participants with Spina Bifida (currently $n=$ 27). Previous data from individuals with SCI/D who played SCI Hard suggested that participants had positive changes on related to self-reports of health behaviors, with no negative consequences noted on measures of emotional health or acceptance of disability. Results for this presentation will examine the evaluations of the game by individuals with Spina Bifida as well as any changes that appeared to occur related to self-reported health behaviors, attitude toward disability, self-efficacy, self-monitoring behavior and quality of life.

Conclusions Electronic games - if thoughtfully designed with the input of the target population - appear to offer a potential avenue for engaging adolescents and young adults with SCI/D in the self-management process. The medium is familiar to them and allows a safe place to learn rules, test boundaries and experiment with the connection between outcomes and actions/inactions.
Estimation of Health State Utility Values for Spina Bifida Using an Online Platform

Inouye, Brian M. ${ }^{1}$, Tejwani, Rohit ${ }^{2}$, Wang, Hsin-Hsiao $^{1}$, Jiang, Ruiyang ${ }^{1}$, Wiener, John S. ${ }^{1}$, Purves, J T. ${ }^{1}$, Routh, Jonathan C. ${ }^{1}$

${ }^{1}$ Duke University Medical Center

${ }^{2}$ Duke University School of Medicine

Background While many Spina Bifida (SB) patients are living well into adulthood, we do not understand the impact of their healthcare on themselves and their families. To perform a cost-utility analysis (CUA), we require condition-specific utility values, numerical representations of disease impact on health-related quality of life (HRQoL). Therefore, we sought to estimate SB utility values through the use of an online time-tradeoff (TTO) instrument.

Methods A TTO instrument was distributed through Amazon's Mechanical Turk online work-distribution interface. Demographic information and prior knowledge of SB were assessed. After being provided a written passage and online video explaining SB and associated comorbidities, participants were queried on hypothetical ascending TTO from a child-parent dyad perspective with a 6-year-old SB child. Respondents also indicated the percentage of time traded from their life vis-à-vis the SB child's. Utility estimates were calculated and compared using bivariate and multivariate analyses.

Results We obtained 503 responses (85\% response rate). Mean respondent age was $34( \pm 11) ; 247$ (49\%) were female; $386(77 \%)$ were white; $189(38 \%)$ were married, and 234 (46\%) had children. Only 51 respondents $(9 \%)$ reported having "good" or "ample" prior knowledge of SB. Eight respondents (0.02\%) had SB. Few had prior experience with SB either in a child (4, $1 \%)$, or friend/relative $(28,5 \%)$. Compared with a perfect health state of 1.0 , we found the mean utility of SB to be 0.8 . Mean proportion of longevity traded by participants in the dyadic interaction was $66 \%( \pm 27)$ from the parent's life.

Conclusions Subjects view SB as significantly inferior to perfect health, despite advances in management options and survivability for this condition and its comorbidities. To continue describing the impact of SB, health state utilities for SB-associated functional impairments and treatment options must be obtained. 